\title{
Endodontic management of fractured crown and rehabitation with fractured natural tooth- All in single visit
}

\author{
Bhaskar Lal \\ PG Student, Awadh Dental College \& Hospital, Jamshedpur, Jharkhand, India
}

Corresponding Author:

Email: bhaskarlal@ymail.com

\begin{abstract}
Introduction: Accidental fracture of upper central incisor are very commonly seen in routine dental practice but management of fractured crown in single sitting are the challenges what we faced in towards practices with rehabilitation with the same natural fractured tooth.

Materials and Methods: This case reported describes the successful endodontic diagnosis and management of fractured upper central incisor and prosthetic rehabilitation with the same fractured crown. Cone beam tomography facilitates the diagnosis of fractured crown.

Results: The upper central incisor with fractured crown with single sitting root canal treatment with placement of post (metal) and core buildup with prosthetic rehabilitation with same fractured natural crown. Cone Beam Computed Tomography and dental operating microscope can facilitate evaluation for teeth and successful treatment.
\end{abstract}

Keywords: Single, Sitting, RCT.

\section{Introduction}

The success of endodontic therapy depends upon thorough knowledge of morphology of tooth. Failure in ecognizing variations in the morphology of tooth and restoration may result in unsuccessful endodontic treatment. Of all teeth treated with primary endodontic therapy and post placement upper anterior tooth are having highest failure rates. This accreditation is primary because of theories variable anatomy and procedures for placement of post. Restoration of fractured anterior tooth with post placement and prosthetic restoration with natural crown with endodontic treatment would be truly challenging. This clinical case report describes the use of Cone Beam Computed Tomography (CBCT) in the successful endodontic management of challenging case.

\section{Case Report}

A $29 \mathrm{yr}$ male patient reported to the department of Conservative dentistry and Endodontic dental college Jamshedpur with the complain of fractured tooth in upper front tooth region with tremendous amount of pain from past 1 day. Patient gives history of trauma to tooth while opening garage metal gate. On clinical examination fractured crown labially to gingival $3^{\text {rd }}$ and palatally CEJ with grade 2 mobility. Tender on percussion was positive.

On radiographic diagnosis evidences of root fracture and or any periapical lesion. On basis of clinical and radiographic diagnosis, the case diagnosis is Acute Irreversible Pulpitis.

Access was gained to the pulp chamber after administration of local anesthesia (2\% lidocaine with 1:80000 adrenaline) to gain sufficient straight line access. The fractured segment was removed. The canal orifice were identified under magnification loop. Canal patency was established with \#10k file. Canal orifice were enlarged and working length was determined using Root zx mini apex locator (j. Morita Tokyo Japan) and later confirmed radiographically. The root canal system was cleaned using denstply pathfile and dentsply wave One Gold rotary canal preparation system using $2.5 \%$ sodium hypochloride and $17 \%$ EDTA was used for $1 \mathrm{~min}$ as the final rinse followed up with saline.

Subsequently canal were obturated with F2 wave one gutta-percha point. (dentsply touch and heat was used for lateral condensation and removal for gutta-percha point from the canal. Metal post was placed and luxa-core dual cure resin was used for post endodontic restoration. Natural fractured crown was modified according to the metal post placed and was cemented to the post using luxa-core dual cure cement. On follow up, after six month, the tooth is asymptomatic and clinically sound.

\section{Summary}

With the advent of technological advancement and emergence of new gadgets, evidence based dentistry and more scientific deliberations and the concept of maximum dentistry in minimum visits led to a resurgent impetus towards laying down of various protocols to enable dentists to venture into single visit endodontics with reasonable level of outcome. Single visit root canal treatment versus the multiple visit root canal treatment has been the subject of a long standing debate within the dental community, when the clinicians are faced with choices of which treatment should be offered to patients, the central issues that should be considered are effectiveness, complication, cost. 


\section{Discussion}

As the need of the patient the crown placement on the same day with the good esthetics result was the challenged faced by the cliniciation. All the treatment have to be done on the same appointment as patient was having job interview on very next day. Seeing to the challenging situation we plan to go for single sitting root canal treatment with post and core placement an prosthetic rehabitation with the same natural crown.
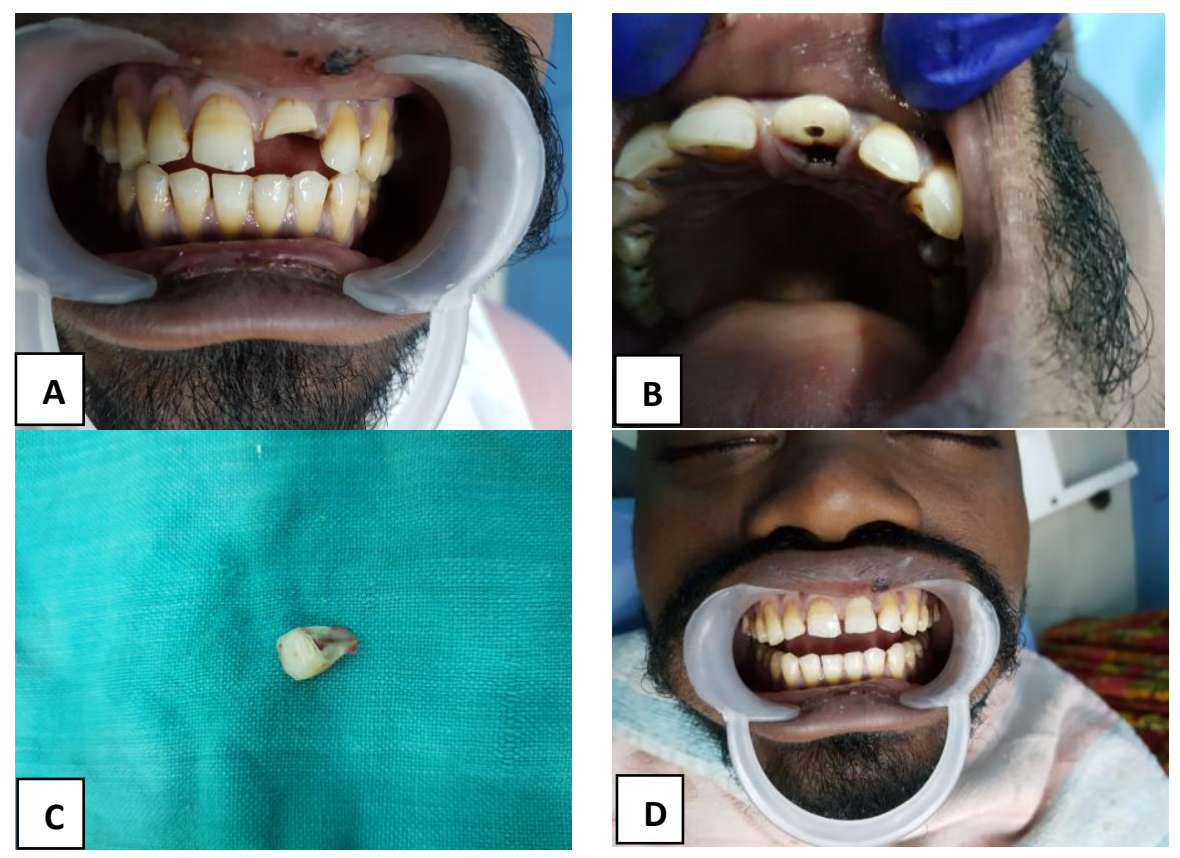

Fig. 1

Fig. 2
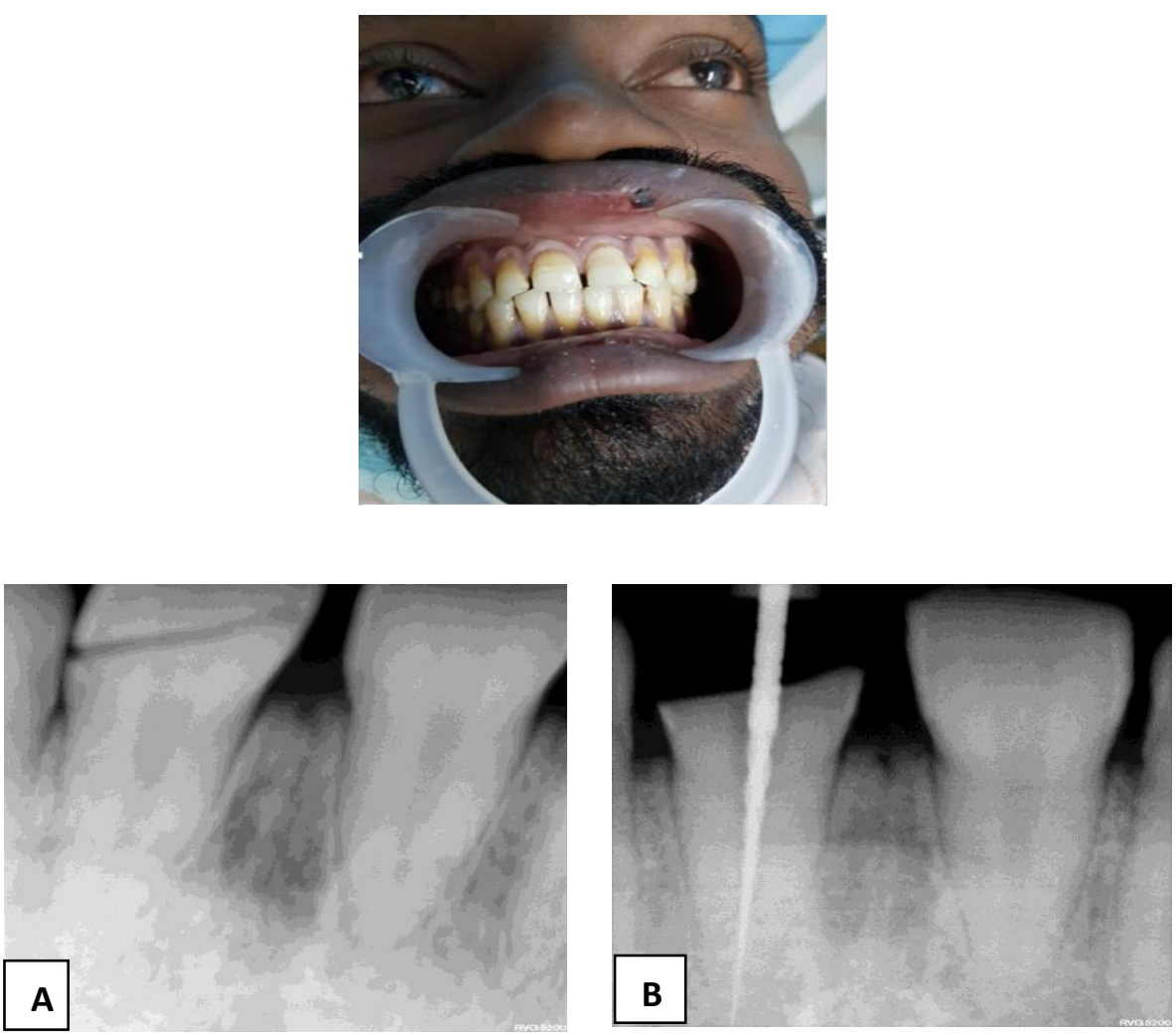

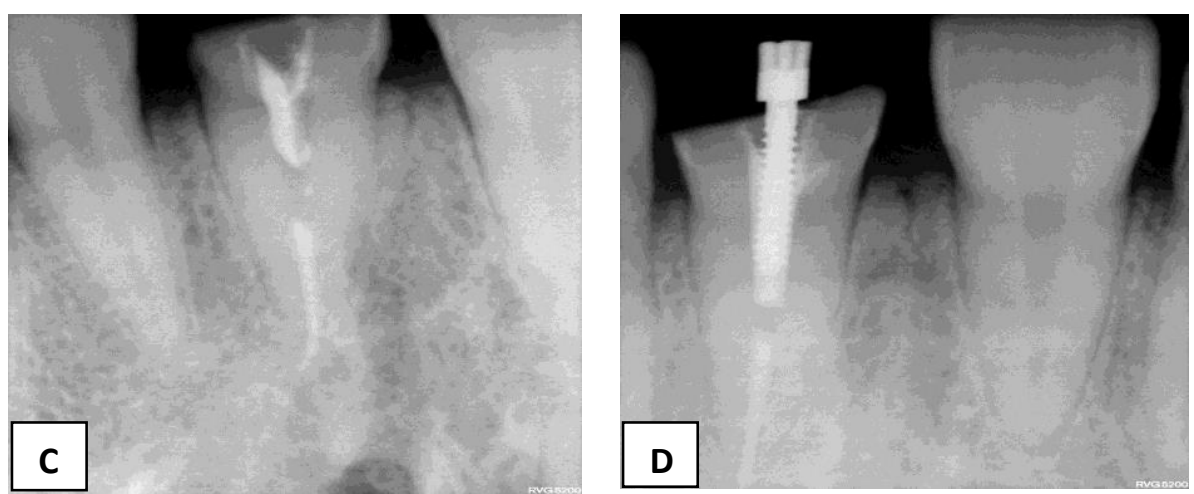

Fig. 3

\section{References}

1. Cohen S, Hargreves KM. Pathways of pulp. Chapter 4: case selection and treatment planning, 9th edition. 2006;94-5.

2. Garg N, Garg A. Text book of Endodontics, Chapter 19: Single visit endodontics, 2nd edition. Jaypee. 2007;301-3.

3. Ashkenaz PJ. One-visit endodontics. Dent Clin North Am. 1984; 28: 853-863. 4. Sathorn C, Parashas P, Messer $\mathrm{H}$. The prevalence of post operative pain and flare up in single and multiple visit endodontic treatment: a systematic review.

4. G et al. Fracture strength of teeth with flared root canals restored with glass fibre posts. International Dental Journal. 2007;57:153-60.

5. Kaira LS et al. Carbon Fiber Post to Reinforce Esthetics. Journal of Dental and Allied Sciences. 2013;2(1):39-42. 